PROCEEDINGS OF THE

AMERICAN MATHEMATICAL SOCIETY

Volume 133, Number 1, Pages $65-70$

S 0002-9939(04)07450-7

Article electronically published on May 12, 2004

\title{
ON A CERTAIN CLASS OF MODULAR FUNCTIONS
}

\author{
WINFRIED KOHNEN
}

(Communicated by Wen-Ching Winnie Li)

\begin{abstract}
We give a characterization of those meromorphic modular functions on a subgroup of finite index of the full modular group whose divisors are supported at the cusps, in terms of the growth of the exponents of their infinite product expansions.
\end{abstract}

\section{IntRoduction AND STATEMENT OF RESUlts}

Let $f$ be a meromorphic modular function of integral weight $k$ on a subgroup $\Gamma$ of finite index in $\Gamma(1):=S L_{2}(\mathbf{Z})$, i.e., $f$ is a meromorphic function on the complex upper half-plane $\mathcal{H}$, satisfies the usual transformation formula in weight $k$, and is meromorphic at the cusps. We will suppose that $f$ is not identically zero. Like any complex-valued, periodic and meromorphic function on $\mathcal{H} \cup\{\infty\}$ that is not identically zero, $f$ then has a product expansion

$$
f(z)=c q_{M}^{h} \prod_{n \geq 1}\left(1-q_{M}^{n}\right)^{c(n)}
$$

where the infinite product is convergent in $\left|q_{M}\right|<\epsilon$ for some $\epsilon>0$. Here $c$ is a nonzero constant, $M$ is the least positive integer with $\left(\begin{array}{cc}1 & M \\ 0 & 1\end{array}\right) \in \Gamma, q_{M}:=e^{2 \pi i z / M}$ for $z \in \mathcal{H}, h$ is the order of $f$ at infinity and the $c(n)(n \in \mathbf{N})$ are uniquely determined complex numbers [1, 2]. As usual, we understand that the complex powers are defined by the principal branch of the complex logarithm.

The purpose of this note is to give a characterization of those forms $f$ that have no zeros or poles on $\mathcal{H}$, in terms of the coefficients $c(n)$. More precisely, we shall prove

Theorem 1. Suppose that $f$ has no zeros or poles on $\mathcal{H}$. Then this assertion is equivalent to the following assertions, respectively:

i) if $\Gamma$ is of finite index in $\Gamma(1)$, then $c(n)<<_{f} \log \log n \cdot \log n \quad(n>2)$ where the constant implied in $<<_{f}$ only depends on $f$;

ii) if $\Gamma$ is a congruence subgroup of $\Gamma(1)$, then $c(n)<<_{f}(\log \log n)^{2} \quad(n>2)$ where the constant implied in $<<_{f}$ only depends on $f$.

Received by the editors February 12, 2003 and, in revised form, July 9, 2003 and October 27, 2003.

2000 Mathematics Subject Classification. Primary 11F11.

(C)2004 American Mathematical Society 
In case $f$ is on $\Gamma_{0}(N)=\left\{\left(\begin{array}{ll}a & b \\ c & d\end{array}\right) \in \Gamma(1)|N| c\right\}$ for some $N \in \mathbf{N}$, one can do much better (at least if $N$ is squarefree) and prove

Theorem 2. Suppose that $\Gamma=\Gamma_{0}(N)$ and $N$ is squarefree. Then $f$ has no zeros or poles on $\mathcal{H}$ if and only if $c(n)(n \in \mathbf{N})$ depends only on the greatest common divisor $(n, N)$.

We think that the assertion of Theorem 2 or a slightly weaker statement eventually would be true for arbitrary $N$, but it seems that this cannot be proved by the method employed here.

Recall that a meromorphic modular function of weight zero with poles and zeros only at the cusps is called a modular unit. Thus if $\Delta$ is the usual discriminant function of weight 12 on $\Gamma(1)$, then $f^{12} / \Delta^{k}$ is a modular unit. It was proved by Kubert and Kubert-Lang (see [5, chap. 4] and the references given there) that on the principal congruence subgroup $\Gamma(N)$ of level $N$ the group of modular units (at least up to 2-torsion) is generated by the so-called Siegel units, which are defined in terms of $N$-division values of the Weierstrass $\sigma$-function. It might be interesting to investigate if Theorem 1, ii) or Theorem 2 could also be proved by the methods applied there.

\section{Proof of Theorem 1}

Put

$$
\theta=q_{M} \frac{d}{d q_{M}}=\frac{M}{2 \pi i} \frac{d}{d z}
$$

Then we have

$$
\frac{\theta f}{f}=h-\sum_{n \geq 1}\left(\sum_{d \mid n} d c(d)\right) q_{M}^{n} \quad\left(\left|q_{M}\right|<\epsilon\right)
$$

(see [1] and [2]).

Therefore, if $c(n)<<_{f} \log \log n \cdot \log n \quad(n>2)$, the right-hand side of (1) is convergent for all $z \in \mathcal{H}$, and hence by standard facts from complex analysis $f$ has no zeros or poles on $\mathcal{H}$.

Now assume the latter condition. Let

$$
P(z)=1-24 \sum_{n \geq 1} \sigma_{1}(n) q^{n} \quad\left(z \in \mathcal{H}, q=q_{1}\right)
$$

be the nearly modular Eisenstein series of weight 2 on $\Gamma(1)$, where $\sigma_{1}(n)=\sum_{d \mid n} d$.

We denote by $\left.\right|_{k}$ the usual slash operator in weight $k$, i.e., for a function $g: \mathcal{H} \rightarrow$ $\mathbf{C}$ and $\gamma=\left(\begin{array}{ll}a & b \\ c & d\end{array}\right) \in G L_{2}^{+}(\mathbf{R})$ we put

$$
\left(\left.g\right|_{k} \gamma\right)(z):=(a d-b c)^{k / 2}(c z+d)^{-k} g\left(\frac{a z+b}{c z+d}\right) \quad(z \in \mathcal{H})
$$

Recall that for $\gamma \in \Gamma(1)$, we have

$$
\left(\left.P\right|_{2} \gamma\right)(z)=P(z)+\frac{12 c}{2 \pi i(c z+d)} \quad(z \in \mathcal{H}),
$$

and hence

$$
\partial f:=12 \cdot \frac{1}{M} \theta f-k P f
$$


is a meromorphic modular function of weight $k+2$ on $\Gamma$ (compare [6, part IV, sect. $5]$ ). Therefore, in view of our assumption, $\frac{\partial f}{f}$ is a meromorphic modular function of weight 2 on $\Gamma$, holomorphic on $\mathcal{H}$.

For all $\gamma \in \Gamma(1)$, we have by $(2)$,

$$
\begin{aligned}
\left.\left(\frac{\partial f}{f}\right)\right|_{2} \gamma & =12 \frac{\left.\frac{1}{M}(\theta f)\right|_{k+2} \gamma}{\left.f\right|_{k} \gamma}-\left.k P\right|_{2} \gamma \\
& =12 \frac{\frac{1}{M} \theta\left(\left.f\right|_{k} \gamma\right)}{\left.f\right|_{k} \gamma}-k P .
\end{aligned}
$$

Since $f$, by assumption, is meromorphic at all cusps, it follows that $\frac{\partial f}{f}$ is holomorphic at all cusps, i.e., $\frac{\partial f}{f}$ is a (holomorphic) modular form of weight 2 on $\Gamma$.

Therefore (cf., e.g., [7] chap. III, sect. 4]) the Fourier coefficients of $\frac{\partial f}{f}$ are $<<_{f}$ $n \log n \quad(n>1)$.

Now recall that

$$
\sigma_{1}(n) \leq 2 n \log \log n
$$

for $n$ large. Indeed, as is well known (cf., e.g., [3] Thm. 323]),

$$
\limsup _{n \rightarrow \infty} \frac{\sigma_{1}(n)}{n \log \log n}=e^{\gamma}
$$

where $\gamma=.57721 \ldots$ is Euler's constant.

Therefore we infer from (1) that

$$
\sum_{d \mid n} d c(d)<<_{f} n \log n \quad(n>1),
$$

and hence, inverting, we find that for $n>2$ one has

$$
\begin{gathered}
c(n) \quad<<_{f} \frac{1}{n} \sum_{d \mid n} d \log d \\
\leq \frac{\log n}{n} \cdot \sigma_{1}(n) \\
<<\log \log n \cdot \log n .
\end{gathered}
$$

This proves i).

To prove ii), assume that $f$ is on the principal congruence subgroup $\Gamma(N)$. Then by [4] the weight 2 modular form $\frac{\partial f}{f}$ is the sum of a cusp form and a linear combination of Eisenstein series, and the Fourier coefficients of the former are $<_{{ }_{f}} n$ and those of the latter for $n \geq 1$ have the shape

$$
\sum_{d \mid n, \frac{n}{d} \equiv \alpha}|d| e^{2 \pi i d \beta / N},
$$

where $\alpha, \beta \in \mathbf{Z} / N \mathbf{Z}$. Hence we find that the Fourier coefficients of $\frac{\partial f}{f}$ are $<<_{f}$ $\sigma_{1}(n)$, and so, by (1),

$$
\sum_{d \mid n} d c(d)<<_{f} \sigma_{1}(n)
$$

which implies that

$$
c(n)<<_{f} \frac{1}{n} \sum_{d \mid n} \sigma_{1}(d)
$$


Arguing in a similar way as before, this proves ii).

\section{Proof of Theorem 2}

We need only prove that if $f$ has no zeros or poles on $\mathcal{H}$, then the numbers $c(n)(n \in \mathbf{N})$ have the asserted property, since the converse statement has already been proved in sect. 2 .

Denote by $M_{k}(N)$ the space of meromorphic modular functions of weight $k$ on $\Gamma_{0}(N)$. Since $N$ is squarefree, the cusps of $\Gamma_{0}(N)$ are represented by the numbers $\frac{1}{t}$ with $t$ running over the positive divisors of $N$.

For $t \mid N$ we let $W_{t}^{N}$ be the Atkin-Lehner involution on $M_{k}(N)$ defined by

$$
g\left|W_{t}^{N}:=g\right|_{k}\left(\begin{array}{cc}
t & \alpha \\
N & t \beta
\end{array}\right)
$$

where $\alpha, \beta \in \mathbf{Z}$ with $t^{2} \beta-N \alpha=t$. The matrix $\left(\begin{array}{cc}t & \alpha \\ N & t \beta\end{array}\right)$ maps the cusp $\infty$ to the cusp $\frac{1}{N / t}$, and hence (up to normalization) the Fourier expansion of $g \mid W_{t}^{N}$ at $\infty$ is the Fourier expansion of $g$ at $\frac{1}{N / t}$.

Furthermore, for $d \mid N$ and $g$ a complex-valued function on $\mathcal{H}$ we set $\left(g \mid V_{d}\right)(z):=$ $g(d z)(z \in \mathcal{H})$.

Then the formulas

$$
g\left|W_{t}^{N}\right| W_{t^{\prime}}^{N}=g \mid W_{t t^{\prime}}^{N} \quad\left(g \in M_{k}(N),\left(t, t^{\prime}\right)=1\right)
$$

and

$$
g\left|V_{d}\right| W_{t}^{N}=g\left|W_{t}^{t}\right| V_{d} \quad\left(g \in M_{k}(t), d \mid \frac{N}{t}\right)
$$

are well known and easily verified.

Let $h_{t}$ be the order of $f^{12}$ at $\frac{1}{N / t}$. Let

$$
\Delta(z)=q \prod_{n \geq 1}\left(1-q^{n}\right)^{24} \quad(z \in \mathcal{H})
$$

be the usual discriminant function of weight 12 on $\Gamma(1)$.

We claim that there are integers $a_{t}(t \mid N)$ and a nonzero integer $a$ such that

$$
F:=\prod_{d \mid N}\left(\Delta \mid V_{d}\right)^{a_{d}}
$$

has order $a h_{t}$ at the cusp $\frac{1}{N / t}$ for all $t \mid N$.

Indeed, if $d, t \mid N$ and $e:=(d, t)$, then by (3) and (4) we find that

$$
\begin{aligned}
\Delta\left|V_{d}\right| W_{t}^{N} & =\Delta\left|V_{e}\right| V_{d / e} \mid W_{t}^{N} \\
& =\Delta\left|V_{e}\right| W_{t}^{t} \mid V_{d / e}
\end{aligned}
$$

and

$$
\begin{aligned}
\Delta\left|V_{e}\right| W_{t}^{t} & =\Delta\left|V_{e}\right| W_{e}^{t} \mid W_{t / e}^{t} \\
& =\Delta\left|V_{e}\right| W_{e}^{e} \mid W_{t / e}^{t} \\
& =e^{-6} \Delta \mid W_{t / e}^{t} \\
& =e^{-6} \Delta \mid W_{t / e}^{t / e} \\
& =t^{6} e^{-12} \Delta \mid V_{t / e},
\end{aligned}
$$


and hence

$$
\Delta\left|V_{d}\right| W_{t}^{N}=\left(\frac{t}{e^{2}}\right)^{6} \Delta \mid V_{d t / e^{2}}
$$

Therefore

$$
F \mid W_{t}^{N}=\prod_{d \mid N}\left(\frac{t}{e^{2}}\right)^{6 a_{d}} \cdot \prod_{d \mid N}\left(\Delta \mid V_{d t / e^{2}}\right)^{a_{d}}
$$

and we find that the order of $F$ at $\frac{1}{N / t}$ is equal to

$$
\sum_{d \mid N} a_{d} \cdot \frac{d t}{e^{2}}
$$

(As a matter of taste, if one wants to formally avoid the use of Atkin-Lehner involutions, to compute the order of $\Delta \mid V_{d}$ at each cusp one can of course argue entirely in terms of local coordinates at the cusps; cf., e.g., [8, Introduction].)

We want to show that the $\left(\sigma_{0}(N), \sigma_{0}(N)\right)$-matrix

$$
A_{N}:=\left(\frac{d t}{e^{2}}\right)_{d|N, t| N}
$$

(where $\sigma_{0}(N)$ is the number of positive divisors of $N$ ) is invertible, which then proves the above claim. We do this by induction on the number of prime divisors of $N$. If $N=1$, there is nothing to prove. Assume that $N=p M$ and $(p, M)=1$. Then, as is immediate from the definitions,

$$
\begin{aligned}
A_{N} & =\left(\begin{array}{cc}
A_{M} & p A_{M} \\
p A_{M} & A_{M}
\end{array}\right) \\
& =\left(\begin{array}{cc}
E & p E \\
p E & E
\end{array}\right)\left(\begin{array}{cc}
A_{M} & 0 \\
0 & A_{M}
\end{array}\right)
\end{aligned}
$$

where $E$ is the unit matrix of size $\sigma_{0}(M)$; hence $A_{N}$ is invertible by the induction hypothesis.

By the valence formula (cf., e.g., 9] Thm. 4.1.4.]) applied to $F$ and by what we proved above, since $\Delta$ does not vanish on $\mathcal{H}$, we have

$$
a \sum_{t \mid N} h_{t}=\frac{k_{1}}{12}\left[\Gamma(1): \Gamma_{0}(N)\right]
$$

where $k_{1}$ is the weight of $F$. On the other hand, by the valence formula applied to $f^{12}$, since $f$ has no zeros or poles on $\mathcal{H}$ by our assumption, we have

$$
\sum_{t \mid N} h_{t}=k\left[\Gamma(1): \Gamma_{0}(N)\right]
$$

Therefore $k_{1}=12 a k$.

The function $\frac{f^{12 a}}{F}$ has weight zero and has no zeros or poles on $\mathcal{H}$ or at the cusps, by what we proved above, and therefore is constant. Since $a$ is not zero, this proves Theorem 2.

\section{ACKNOWLEDGMENTS}

The author is very much indebted to M. Knopp and S. Robins for a useful discussion. He is also very grateful to the referee for useful comments. 


\section{REFERENCES}

1. J. H. Bruinier, W. Kohnen and K. Ono: The arithmetic of the values of modular functions and the divisors of modular forms, to appear in Compositio Math.

2. W. Eholzer and N.-P. Skoruppa: Product expansions of conformal characters, Phys. Lett. B. 388 (1996), 82-89. MR $97 \mathrm{k}: 81132$

3. G. H. Hardy and E. M. Wright: An Introduction to the Theory of Numbers, Oxford University Press, Oxford, 1975. MR 81i:10002

4. E. Hecke: Theorie der Eisensteinschen Reihen höherer Stufe und ihre Anwendung auf Funktionentheorie und Arithmetik, In: Erich Hecke, Mathematische Werke (ed.: Akademie der Wissenschaften Göttingen), pp. 461-486, Vandenhoeck \& Ruprecht: Göttingen, 1959. MF 21:3303

5. D. S. Kubert and S. Lang: Modular units, Grundlehren der Mathematischen Wissenschaften, no. 244, Springer-Verlag, Berlin, Heidelberg, New York, 1981. MR84h:12009

6. S. Lang: Introduction to Modular Forms, Grundlehren der Mathematischen Wissenschaften, no. 222, Springer-Verlag, Berlin, Heidelberg, New York, 1976. MR.55:2751

7. H. Maass: Lectures on Modular Functions of One Complex Variable, Tata Institute of Fundamental Research, Bombay, 1964 (revised 1983). MR85g:11034

8. Y. Martin: Multiplicative $\eta$-quotients, Trans. Amer. Math. Soc. 348, no. 12 (1996), 4825-4856. MR97d:11070

9. R. A. Rankin: Modular Forms and Functions, Cambridge University Press, Cambridge, 1977. $\operatorname{MR} 58: 16518$

Mathematisches Institut, Universität Heidelberg, INF 288, D-69120 Heidelberg, GerMANY

E-mail address: winfried@mathi.uni-heidelberg.de 